\title{
Breaking the 100-fs barrier with a streak camera
}

\author{
P. A. Jaanimagi* \\ Laboratory for Laser Energetics, University of Rochester \\ 250 East River Road, Rochester, NY 14623-1299
}

\begin{abstract}
We present a novel x-ray streak tube design that uses a modest extraction field at the photocathode, axial time-of-flight dispersion compensation, and transverse energy selection to achieve better-than-100-fs time resolution.
\end{abstract}

Key words: Streak camera, ultrafast detector

\section{INTRODUCTION}

Historically the time resolution of optical detectors has kept pace with the time duration of pulsed sources, but in 1949, Courtney-Pratt's invention of the streak camera ${ }^{1}$ allowed the diagnosis of events in the nanosecond regime, shifting the balance far toward the detector side. In the 1960s shortly after the discovery of the laser, however, the capability to produce ultrashort pulses had surpassed the direct measurement of their duration and remains so today. Not until 1973, with Bradley's introduction of an extraction-mesh electrode to increase the electric field at the photocathode, ${ }^{2}$ did the time resolution of a streak camera improve to the picosecond regime. A demonstration of subpicosecond time resolution was published in 1975. ${ }^{3}$ This time resolution was available only near the long-wavelength cutoff of the photocathode sensitivity. The current state of the art for optical cameras of 300 to $500 \mathrm{fs}$ was reached in the late 1980s ${ }^{4-6}$ Meanwhile x-ray streak camera time resolution remained at 5 to $10 \mathrm{ps}^{7,8}$ until pushed to the 2-ps level by the capability to produce picosecond $\mathrm{x}$-ray pulses in 1990. ${ }^{9}$ Since then, x-ray time resolution has been demonstrated at the 500 to 600 fs level. ${ }^{10-15}$ Still, the 10 -fs instrumental resolution predicted by Zavoisky and Fanchenko in 1965 remains elusive. ${ }^{16}$ In this paper we will review the many issues that need to be resolved to break the 100-fs time resolution barrier and present a conceptual tube design that calculations predict will achieve better than 50 fs.

\section{BACKGROUND}

Streak cameras operate on the principal of producing an electron analog of the optical or x-ray signal with a photocathode. An electron lens is then used to focus the electrons to an image plane, typically a phosphor screen, but while in transit, the electron beam is deflected to convert the time dimension to a spatial dimension. The streaked image is recorded with a highsensitivity, yet-straightforward photographic system. The limitation in time resolution can be stated as follows: electrons emitted at the same time from a tiny spatial resolution element on the photocathode do not converge to a single point in the image plane nor arrive at the same time. The static point spread function (PSF) of your typical electrostatically focused streak tube is dominated by the aperture aberrations of the electron optics, specifically third-order spherical. This enters into the technical time resolution defined as

$$
\tau_{t}=\omega / v_{\mathrm{st}},
$$

where $\omega$ is the FWHM of the PSF and $v_{\mathrm{st}}$ is the streak speed. The potential for good time resolution is limited to the paraxial region at best. Chromatic aberrations due to the finite spread in the initial electron energies $\varepsilon$ and their angular distribution

*uhxr@1le.rochester.edu; phone 1585 275-5101; fax 1585 275-5960 
ensure that a $\delta$-function pulse of electrons emitted from the photocathode does not arrive at the screen simultaneously. The time dispersion due to the time-of-flight can be separated into the three Cartesian components as $\tau_{\varepsilon}=f\left(\varepsilon_{x}, \varepsilon_{y}, \varepsilon_{z}\right)$, where $x$ and $y$ are the transverse components in the spatial and temporal directions, respectively, and $z$ is along the electron optic axis. The general form up to order $\varepsilon$ may be written as

$$
\begin{aligned}
\tau_{\varepsilon x} & = \pm T_{1 x} \times X_{0} \times \sqrt{\varepsilon_{x}}+T_{2 x} \times \varepsilon_{x}, \\
\tau_{\varepsilon y} & = \pm T_{1 y} \times Y_{0} \times \sqrt{\varepsilon_{y}}+T_{2 y} \times \varepsilon_{y} \\
& = \pm S_{1 y} \times Y_{\mathrm{vc}} \times \beta+S_{2 y} \times \beta^{2}, \\
\tau_{\varepsilon z} & =-T_{1 z} \times \sqrt{\varepsilon_{z}}+T_{1 z e} \times \varepsilon_{z}-T_{2 z} \times \varepsilon_{z},
\end{aligned}
$$

where for the transverse components, the \pm sign is used if the initial velocity is directed away from/toward the axis, and $\left(X_{0}, Y_{0}\right)$ is the initial location at the photocathode. In this report $\tau_{\varepsilon}$ is in picoseconds, $X_{0}$ and $Y_{0}$ are in millimeters and $\varepsilon$ is in electron volts. When a slot is used for the acceleration electrode, it may be more convenient to calculate $\tau_{\varepsilon y}$ in terms of $\beta$, the angle the trajectory makes at the screen with the central ray from the same cathode point, and $Y_{\mathrm{vc}}$, the location at the virtual cathode. ${ }^{17}$ The axial component $\tau_{\varepsilon z}$ has received the most attention, but when pushing the limits to $<100 \mathrm{fs}$, the transverse components also become important. The process of deflecting the electron beam introduces both a spatial distortion and a temporal dispersion onto the signal, which can be lumped together as $\tau_{d}$. Finally, we can have a spatial and temporal distortion due to the space charge of the electron packet with itself during transit from the cathode to the screen $\tau_{\mathrm{sp}}$. The system response is then the convolution of these four components, $\tau_{\mathrm{sys}}=f\left(\tau_{t}, \tau_{\varepsilon}, \tau_{d}, \tau_{\mathrm{sp}}\right)$. Since the components are interrelated, they cannot be simply added in quadrature.

It can be shown easily that as long as the electron optic axis is straight, electrons that start on axis with finite axial energy will arrive at the screen before electrons emitted with less initial axial energy, independent of the axial potential distribution. Yet, for the last few decades, the main effort to improve streak camera time resolution has been concentrated on minimizing this time-of-flight dispersion with what may be termed the "brute force" method. One increases the dc electric field at the photocathode to just below breakdown, around $6 \mathrm{kV} / \mathrm{mm}$, and then resorts to pulsed-mode operation where fields of $25 \mathrm{kV} / \mathrm{mm}$ can be maintained for nanoseconds. ${ }^{12,18}$ This process has resulted in the current state of the art, but future improvements are doubtful since it ignores the time dispersion in the region from the extraction electrode to the deflection plates. We may also assume that a magic x-ray photocathode material with a negligibly small electron energy distribution will not become available, and that we continue to use the standard secondary electron energy distribution curves (SE-EDC) published by Henke et al. ${ }^{19}$ The usual X-ray photocathode materials of $\mathrm{KBr}, \mathrm{KI}$, and gold will be considered in this report. The SE-EDC for $\mathrm{KBr}$ is presented in Fig. 1. We also include the axial component and one transverse component, calculated assuming a Lambertian angular distribution. These distributions are used with Eq. (2) to evaluate the time-of-flight dispersion. To make further progress we must consider alternative techniques such as incorporating a spectrometer into the tube design to select a narrow range of electron energies, or using a curved electron optic axis to correct the transit time of the electrons, compensating for their initial velocities. As will be shown later, $<100$-fs designs must incorporate all of the above techniques.

There are some notable examples of innovation in tube design that have addressed the above issues. The bilamellar streak tube designs from the French, first published in $1974,{ }^{20}$ eliminated the low-voltage focusing section of the classic "stack of cylindrical tubes" design. ${ }^{14,21}$ Electrons were accelerated directly to the anode potential with only a minimal reduced voltage section for focusing. This decreased the time-of-flight dispersion and improved any space-charge-induced 


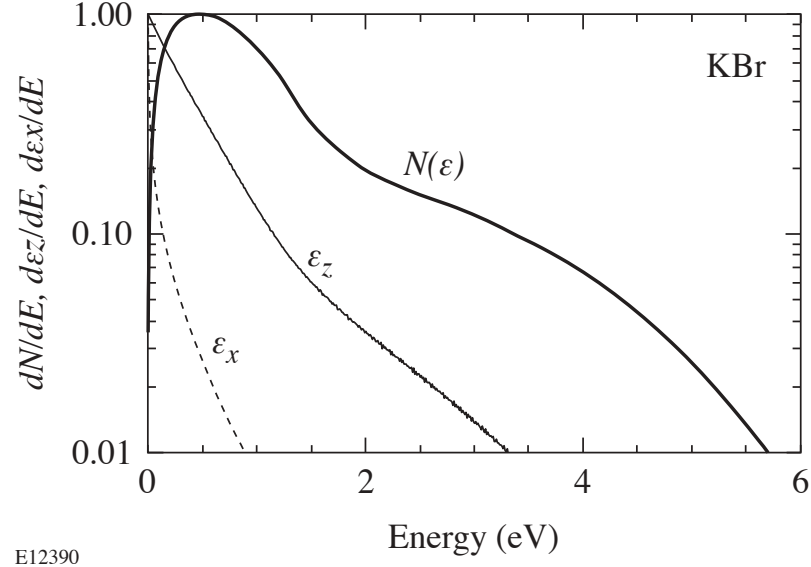

Figure 1: The secondary electron energy distribution for $\mathrm{KBr}$ from Henke et al. ${ }^{19}$ is used to calculate the axial component and one transverse component assuming a Lambertian angular distribution.

distortions. The focusing in the spatial direction was dramatically improved with the quadrupole in this design; however, the 1-D cylindrical lenses that are still used to focus the beam in the time direction have large aberrations and therefore required a limiting aperture in front of the deflection plates to reject the skew trajectories. The size of this aperture has been further reduced and now is also used to filter out electrons with a large $\tau_{\varepsilon y}$ component and thereby improve the time resolution. ${ }^{12}$

In 1976, Lieber et al. 7,22 reported on their novel proximity focused streak tube design. The dc extraction field was brute forced to a passive microchannel plate collimator. This collimator reduced the distance to the deflection plates to its own thickness (about $1 \mathrm{~mm}$ ) and filtered out electrons with large transverse energies. The tube had very limited success due to a poor technical time resolution, although $\tau_{t}$ could be improved by increasing the collimator thickness with the corresponding reduction in throughput.

The magnetically focused tube design published by Kinoshita in $1987^{4}$ had the distinct advantage that the time-of-flight dispersion due to the transverse energy components reduced to zero on axis. This tube design also accelerated the electrons directly to the anode potential and minimized the distance from the extraction mesh to the deflection plates.

In 1988 Niu et al..$^{23}$ published the only streak tube design that attempted to curve the optic axis, as the "half-open aperture" tube. Realizing that there is a well-defined temporal shear in the $y$ direction across the spatial profile of the beam at the anode, an aperture was used to select an off-axis portion of the beam such that the shear partially corrected the axial time dispersion. A further correction was then applied by using the temporal shear produced by a static voltage on a pair of deflection plates (referred to as the compensation element). This deflection curved the optic axis prior to the beam reaching the streaking deflection plates. Unfortunately no results implementing these two concepts have been published.

\section{FOCUSING ABERRATIONS}

Achieving $<100$-fs time resolution demands that particular attention be paid to the quality of the electron lenses used in the streak tube. Not only is a tight spatial focus required for the technical time resolution, but also the time-of-flight dispersion through the lens from the transverse chromatic aberrations must be minimized; if not, it will dominate the system resolution. Recall that even a vacuum is dispersive for electron velocities. A comparison of the quality of spatial focus that can be obtained with different electron lenses is presented in Fig. 2. These plots are from 2- and 3-D electron optics codes that solve the Laplace equation and calculate electron trajectories and equipotential surfaces. The problem represented has a $15 \mathrm{kV} / 3-\mathrm{mm}$ mesh extraction field at the cathode followed by a constant $15 \mathrm{kV}$ potential to the screen. The nominal spatial 

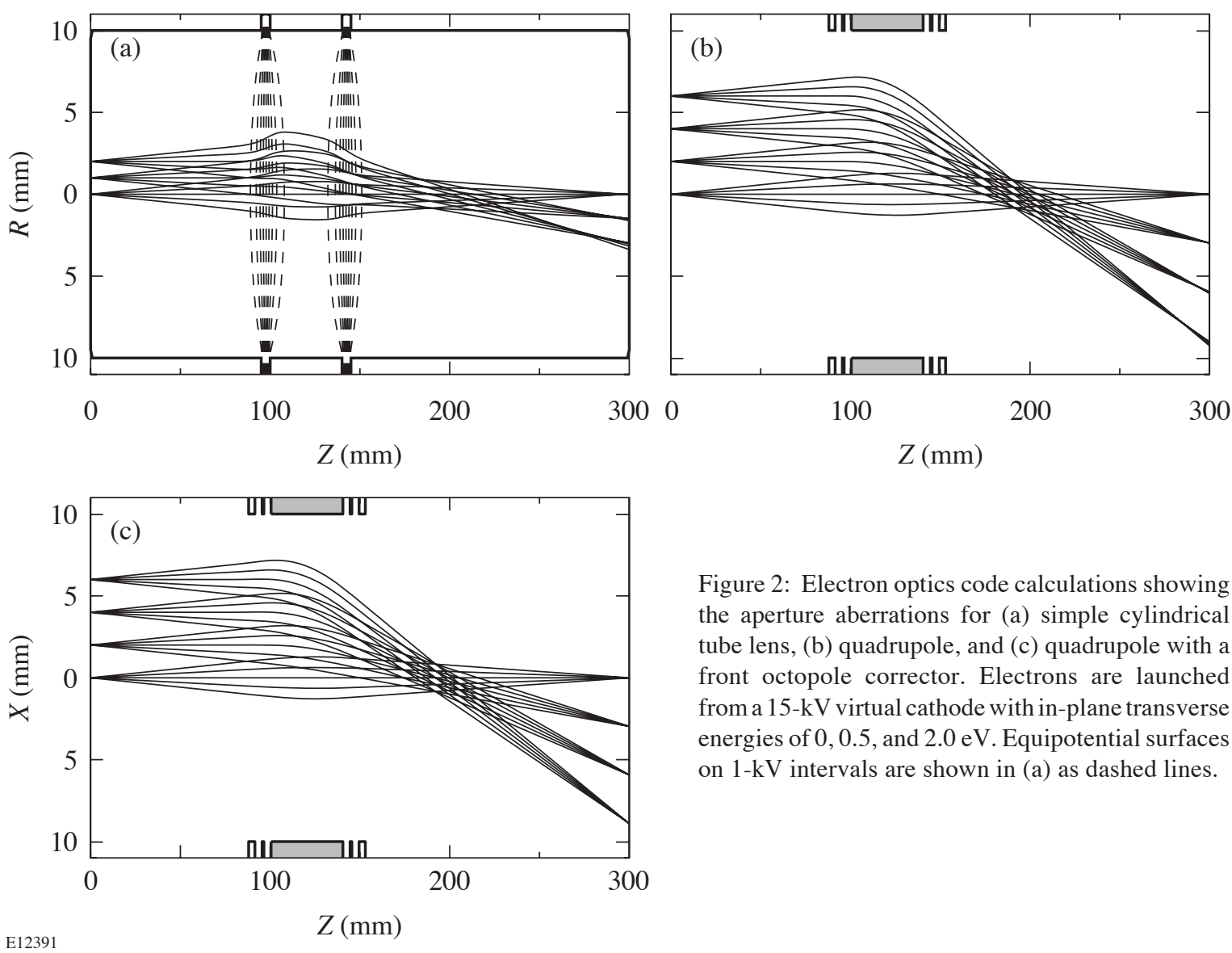

Figure 2: Electron optics code calculations showing the aperture aberrations for (a) simple cylindrical tube lens, (b) quadrupole, and (c) quadrupole with a front octopole corrector. Electrons are launched from a $15-\mathrm{kV}$ virtual cathode with in-plane transverse energies of $0,0.5$, and $2.0 \mathrm{eV}$. Equipotential surfaces on $1-\mathrm{kV}$ intervals are shown in (a) as dashed lines.

magnification is 1.5 . The axial potential decreases to $5416 \mathrm{~V}$ in the central focusing section of the cylindrical tube lens of Fig. 2(a) as shown by the equipotential lines but maintains at $15 \mathrm{kV}$ for the quadrupole lens of Fig. 2(b). We launch electrons from the $15 \mathrm{kV}$ virtual cathode behind the true photocathode with in-plane transverse energies of $0,0.5$, and $2.0 \mathrm{eV}$.

The tube lens in Fig. 2(a) has a good spatial PSF over the central region, $X_{0}< \pm 1.0 \mathrm{~mm}$; the lens fill factor is $<10 \%$. Outside of this region, third-order spherical aberrations cause the off-axis focus to be well in front of the screen. A curved screen to compensate for the parabolic image plane is too tightly curved to be practical. The 1-D version of this tube lens that is used in the bilamellar tubes for focusing in the temporal direction has comparable aberrations. One must also limit its use to $<10 \%$ fill factor, which becomes an issue when a slot is used for the extraction electrode because the beam is more divergent than if a mesh were used. The quadrupole lens in Fig. 2(b) has superior imaging properties, which can be further improved with a front octopole corrector ${ }^{24}$ as shown in Fig. 2(c). The lens fill factor now approaches $80 \%$.

The details of the time-of-flight through the focusing lens are presented in Fig. 3 for the tube lens and the corrected quadrupole. The abscissa $\theta_{f}$ is the angle the trajectories make with the screen, and the ordinate is the transit time relative to a zero-energy electron emitted on axis. The solid lines denote the streak curvature. Curvature of the isotemporal lines due to the longer transit times for off-axis points may be problematic for the short streak durations $(<20 \mathrm{ps})$ that are generally used for subpicosecond time resolution. The streak curvature can be written as a parabolic function of the initial cathode position. The tube lens has $\Delta \tau(\mathrm{ps})=0.698 \times X_{0}^{2}(\mathrm{~mm})$, and the quadrupole has $\Delta \tau(\mathrm{ps})=0.313 \times X_{0}^{2}(\mathrm{~mm})$. Both tube designs can be corrected easily by using a curved fiber-optic faceplate for the screen substrate. 


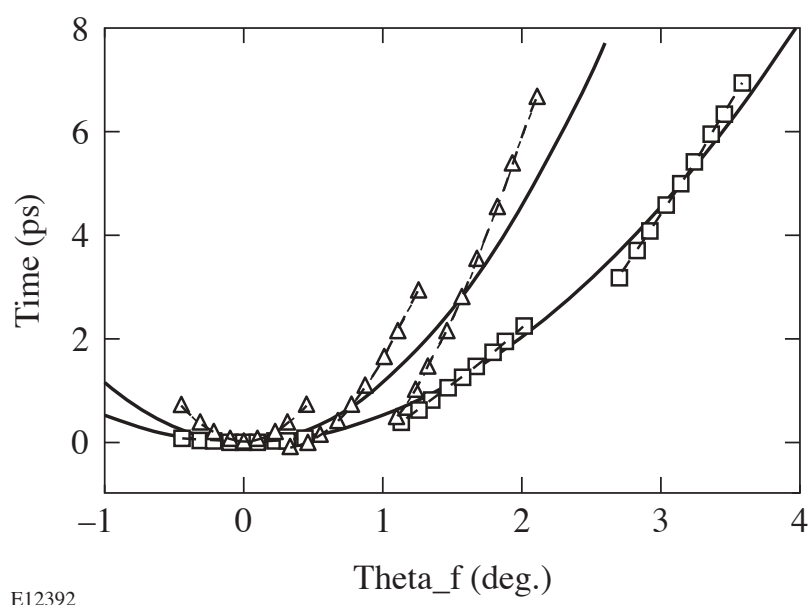

Figure 3: Time-of-flight dispersion for the electron lenses in Figs. 2(a) and 2(c) as a function of the angle the trajectory makes with the screen. The solid curves are the isotemporal lines. Electrons are launched from $X_{0}=0,1$, and $2 \mathrm{~mm}$ for the tube lens (triangles) and $X_{0}=0,2$, and $4 \mathrm{~mm}$ for the quadrupole (squares). The initial electron transverse energies from each point are $0.0, \pm 0.1, \pm 0.5, \pm 1.0$, and $\pm 2.0 \mathrm{eV}$. The time resolution - the spread about the isotemporal lines degrades quickly for points off-axis.

The time dispersion is the spread in transit times about the isotemporal line and is shown for $\left|\varepsilon_{x}\right| \leq 2 \mathrm{eV}$, with initial positions of $X_{0}=0,1$, and $2 \mathrm{~mm}$ for the tube lens (triangles), and $X_{0}=0,2$, and $4 \mathrm{~mm}$ for the quadrupole (squares). The dispersion coefficients obtained by fitting the data to $\tau_{\varepsilon x}$ from Eq. (2) are $T_{1 x}=1.09$ and $T_{2 x}=0.345$ for the tube lens example, and $T_{1 x}=0.329$ and $T_{2 x}=0.029$ for the quadrupole. The $T_{1 x}$ term dominates the PSF in time for these examples. The transverse energy $\varepsilon_{x}$ limit to obtain a time resolution $<50$ fs at $X_{0}=1 \mathrm{~mm}$ can be determined as $\sqrt{\varepsilon_{x}}<0.025 \mathrm{ps} / T_{1 x}$, or a few meV. For such small $\varepsilon_{x}$ values, the $T_{2 x}$ term can be ignored. Fortunately the crossover point offers an ideal location to limit $\varepsilon_{x}$ since at this location the transverse velocities are neatly dispersed in space [see Fig. 2 (c)] at $z=190 \mathrm{~mm}$. Of course, clipping the larger $\varepsilon_{x}$ values will reduce the signal throughput. There is a similar issue with the time-of-flight dispersion from $\varepsilon_{y}$; the beam divergence in the $y$ direction must also be severely restricted. The scaling of the transverse chromatic aberration coefficients with the beam potential is $T_{1 x} \propto V_{a}^{-1}, T_{2 x} \propto V_{a}^{-1.5}$. Everything improves with higher beam potential.

\section{DEFLECTION SYSTEM}

There is a recurring theme among the reported subpicosecond cameras: the deflection plates must be a traveling-wave system, typically a meander line. This is due to the limited bandwidth of a simple two-plate deflector. The other advantage of the meander line is the increased deflection sensitivity. Further, one can reduce the fringing fields by placing ground planes between the lines. Still, the aberrations inherent in deflecting the beam remain (we are not aware of any reports showing otherwise). These aberrations can be illustrated with the static field parallel-plate deflector shown in Fig. 4. The deflection angle $\alpha(\mathrm{rad})$ can be written as

$$
\alpha=L V_{d} / 2 d V_{a}
$$

where $L$ is the plate length, $d$ is their separation, $V_{d}$ is the total voltage on the plates, $V_{a}$ is the anode or beam potential, and we ignore relativistic effects. The fringing field contributes about $50 \%$ more deflection. When a beam of dimension $2 w$ traverses the field between the plates, the top part of beam will be at a different potential than the lower part. This difference in axial velocities introduces a time shear across the beam given approximately by

$$
\tau_{d}=w L V_{d} / v_{a} d V_{a}=2 \alpha w / v_{a}
$$

where $v_{a}$ is the average electron axial velocity. Here we consider only the region delimited by the plate length, whereas in practice the fringing fields will increase the time dispersion. The time shear changes sign as the beam crosses the axis, resulting in a minimum in the time dispersion near the axis. ${ }^{25}$ With a meander line deflection system, each segment 


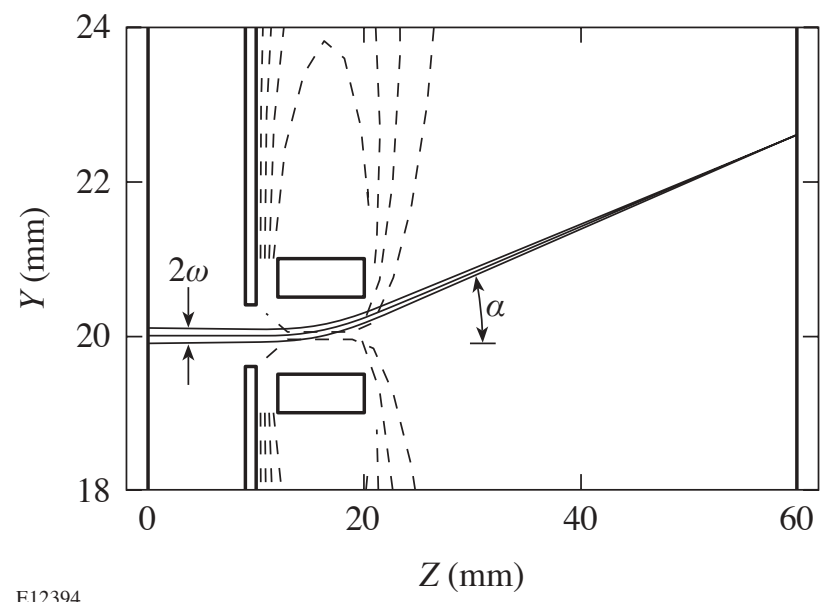

Figure 4: Simple parallel plate deflection system with static $\pm 200-\mathrm{V}$ potentials and a $16-\mathrm{keV}$ electron beam. The dashed lines are equipotentials showing the fringing fields.

contributes a little to the deflection angle and to the time dispersion, but Eqs. (3) and (4) will still give the total angle and time dispersion. Any reduction in the fringing fields will affect both the angle and the time dispersion.

Restricting $\tau_{d}$ to $<50$ fs limits the usable streak length on the screen to \pm a few millimeters. Typical parameter values would be $\alpha=1.0^{\circ}, w=0.1 \mathrm{~mm}, V_{a}=15 \mathrm{kV}$, and a $100-\mathrm{mm}$ deflection distance. These requirements on the beam size/divergence are commensurate with limiting the time-of-flight dispersion due to $\varepsilon_{y}$. Again, increasing the beam potential is beneficial to reduce $\tau_{d}$ although it will decrease the deflection sensitivity. Any beam focusing by the deflection system is not an issue for the small angles that one is limited to here.

\section{MIRRORED SYSTEMS}

The time-of-flight dispersion coefficients due to the electrons' axial energy distribution from Eq. (2) are well known as

$$
\begin{aligned}
& T_{1 z}=k / E_{\mathrm{ex}}, \\
& T_{1 z e}=T_{1 z} / 2 V_{\mathrm{ex}}^{1 / 2}, \\
& T_{2 z}=k L_{2} / 4 V_{e x}^{3 / 2},
\end{aligned}
$$

where $k=(2 m / e)^{1 / 2}=3.3724 \times 10^{-6}$ in MKS units, $E_{\mathrm{ex}}$ is the extraction field at the photocathode, $V_{\mathrm{ex}}$ is the extraction potential, and $L_{2}$, is the distance from the extraction electrode to the deflection plates. The $T_{2 z}$ term assumes that the potential is constant over $L_{2}$, otherwise, the integral over the axial potential distribution must be used. The axial time-offlight dispersion for a $16 \mathrm{kV} / 4$-mm extraction field and $L_{2}=30$-cm tube design is plotted in Fig. 5 (a) for three typical x-ray photocathodes. We assume a Lambertian angular distribution and ignore the transverse components for now. The negative dispersion $\tau_{\varepsilon z}(\mathrm{ps})=-0.8431 \sqrt{\varepsilon_{x z}}-0.1216 \varepsilon_{z}$ limits the time resolution to about $0.75 \mathrm{ps}$.

A normal-incidence electron mirror is the simplest technique to circumvent the straight electron optic axis conundrum. The round-trip time-of-flight dispersion for a mirror is given as

$$
\tau_{m z}=T_{3 z} \times \varepsilon_{z}=\left(k / E_{m} V_{\mathrm{ex}}^{1 / 2}\right) \times \varepsilon_{z}
$$



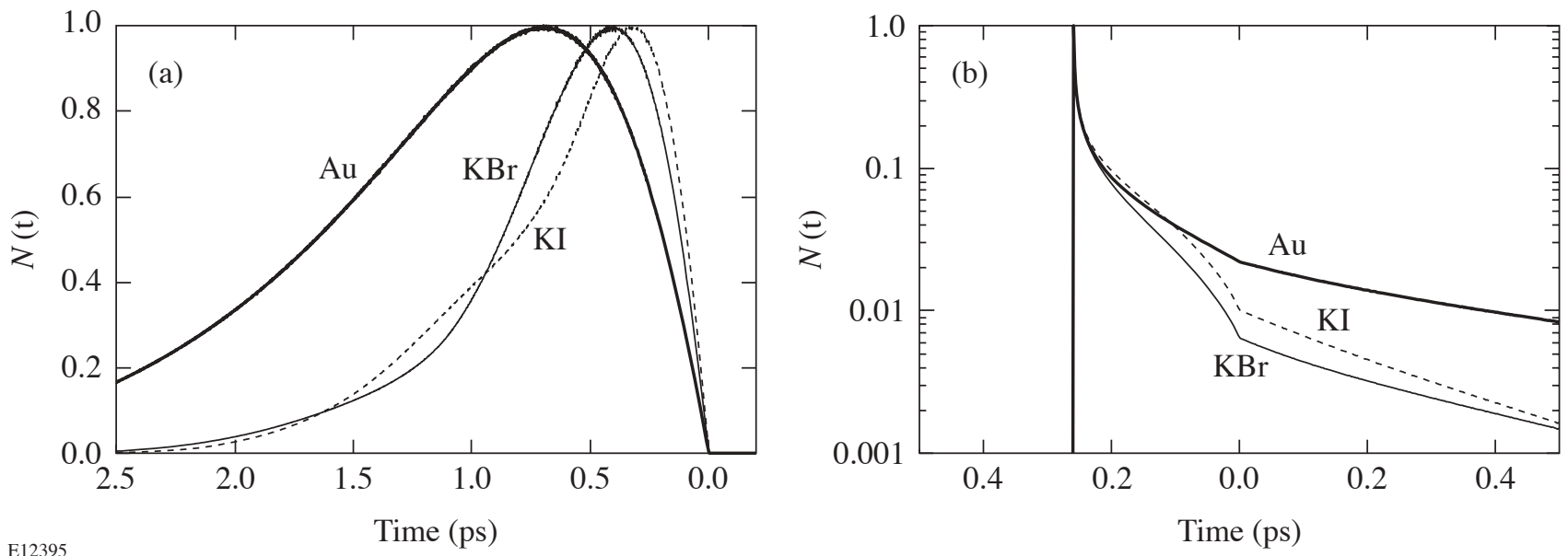

Figure 5: (a) The axial time-of-flight dispersion calculated for $\mathrm{KI}, \mathrm{KBr}$, and $\mathrm{Au} \mathrm{x}$-ray photocathode materials for a 16-kV/4-mm extraction field followed by a $30-\mathrm{cm}$ focusing region at $16-\mathrm{kV}$ potential. A Lambertian angular distribution is assumed. Time resolution is $750 \mathrm{fs}$. (b) A positive time dispersion of $0.8 \mathrm{ps} / \mathrm{eV}$ from an electron mirror partially corrects the negative time dispersion in (a) and improves the time resolution to $10 \mathrm{fs}$.

where $E_{m}$ is the electric field of the mirror. The positive time dispersion provided by the mirror can be used to cancel the $T_{2 z}$ term and partially compensate the $\mathrm{T}_{1 z}$ term. A mirror with $E_{m}=E_{\mathrm{ex}} / 120$ will provide a $T_{3 z}$ term of $+0.8 \mathrm{ps} / \mathrm{eV}$ that improves the time resolution to the $10 \mathrm{fs}$ range as shown in Fig. 5 (b). Even the full width at the $10 \%$ level is $<100 \mathrm{fs}$. There are, however, two problems with this scheme. First, one must be concerned with the space-charge-induced broadening as the beam is brought to a stop at the turnaround point. Second, the return beam intersects the cathode. Rempfer ${ }^{26}$ has published an image-relayed electron optic system that incorporates a magnetic field perpendicular to the beam-propagation direction to bend the electrons into (and, upon return, out of) a normal-incidence mirror. It is not clear whether such a system can be incorporated into a streak camera.

The next-simplest electron mirror is a corner cube as shown in Fig. 6 . Now the turnaround point is at potential $V_{a} / 2$, which is good for any space-charge issues; however, the dispersion for this mirror is negative:

$$
\tau_{\mathrm{ccz}}=-\left[k s /\left(2 V_{a}\right)^{3 / 2}\right] \times \varepsilon_{z}
$$

where $s$ is distance from the corner to where the $\varepsilon_{z}=0 \mathrm{eV}$ trajectory exits or enters the mirrors. The higher-energy electrons travel a shorter diagonal distance across the corner, thereby canceling the positive dispersion gained in the mirror. Additional problems with a corner cube mirror are the poor throughput traversing four meshes at $45^{\circ}$, the difficulty of capping the ends with a linear gradient, and the focusing power for divergent beams.

\section{DOUBLE CYLINDRICAL ANALYZER}

The cylindrical analyzer formed with concentric cylinders has dispersive and focusing properties that can be used to form the front end of a streak camera. The potential between the cylinders is given by

$$
V(r)=V_{i}+\left(V_{o}+V_{i}\right) \ln \left(r / r_{i}\right) / \ln \left(r_{o} / r_{i}\right),
$$

where the subscripts refer to the inner and outer cylinders. Typically the centerline potential is set to the beam potential, $V_{b}$. The radial gradient can be varied independently and is used to tune the dispersive properties. An ideal cylindrical analyzer 
is modeled in Fig. 7. Electrons that are started from the centerline with equal energy but with a range of angles converge at the first focus, $127^{\circ}$ around the arc, and then again at $254^{\circ}$ with negligible time dispersion. Electrons that are started tangential to the centerline with increasing energy follow longer paths (introducing positive axial dispersion) but converge at the second focal position. The magnitude of the dispersion $d \tau / d \varepsilon_{z}$ can be varied easily. It is proportional to the cylinder radius. It scales with the beam potential as $V_{b}^{-3 / 2}$, and as the voltage gradient is varied, it scales inversely with the potential at the first focus. The arc length to the first and second focus increases by a few degrees as the beam potential and the gradient are increased due to relativistic effects.

A realistic implementation of the cylindrical analyzer as the front end of a streak tube is presented in Fig. 8. The first issue is capping the ends of the cylinders in a manner allowing the addition of a photocathode while preserving as much of the dispersive properties as possible. The extraction electrode at the centerline potential forms the end cap; either a slot or a mesh may be used. The ends of the cylinders are folded inward a small bit to minimize the end effects. The top and bottom of the cylinders (spatial or $x$ direction) are capped similarly at a total height at least four times the difference in cylinder radii. The two cylinders are joined at the second focus of the ideal analyzer shown in Fig. 7. The axial time-of-flight dispersion for the system is $\tau_{\varepsilon z}=-0.906 \times \sqrt{\varepsilon_{z}}+0.861 \times \varepsilon_{z}$, which by itself results in a time response similar to Fig. 5(b). An interesting property of the double cylinder is that the magnitude of the $T_{2 y}$ term scales with the positive axial dispersion term; in this example, $T_{2 y}=0.825$.

A mesh extraction electrode is not recommended for optimum performance of this tube design. Electrons launched from a point on the photocathode with equal energy but a range of angles enter the cylindrical section at different radii along the

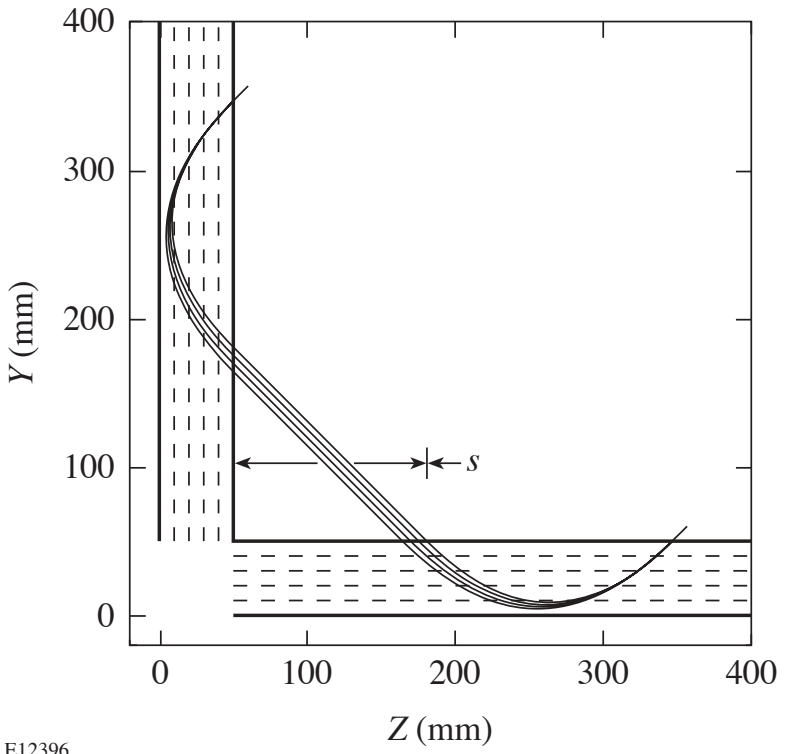

E12396

Figure 6: An electron optic corner cube mirror.

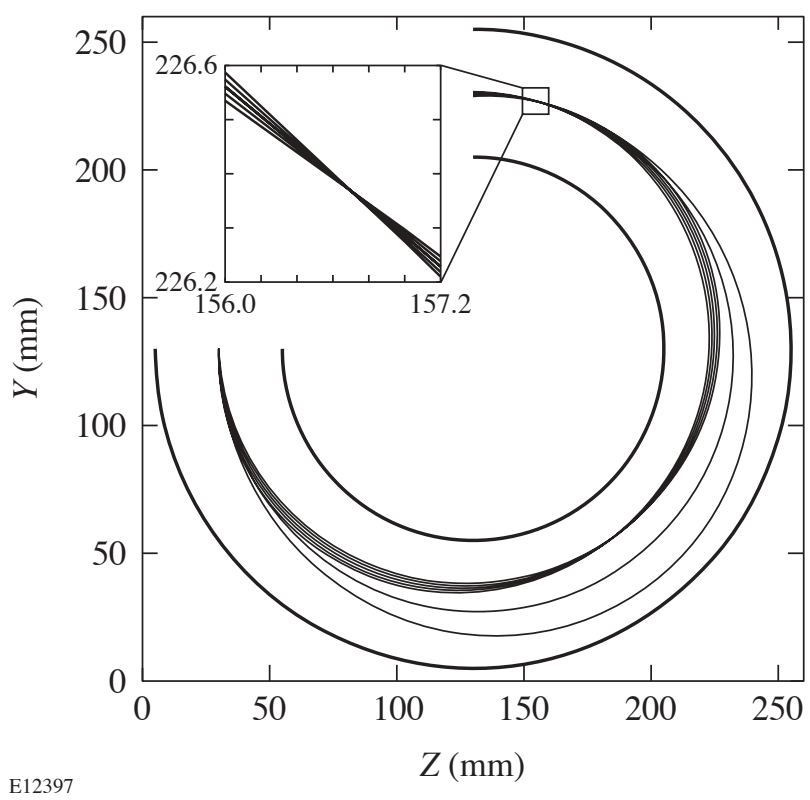

Figure 7: An ideal cylindrical analyzer introduces positive axial dispersion. Electrons that are started from the centerline with equal energy but a range of angles converge at the first focus, $127^{\circ}$ around the arc, and then again at $254^{\circ}$ with negligible time dispersion. Electrons that are started tangential to the centerline with increasing energy follow longer paths but converge at the second focal position. The inset shows the very tight second focal position. 


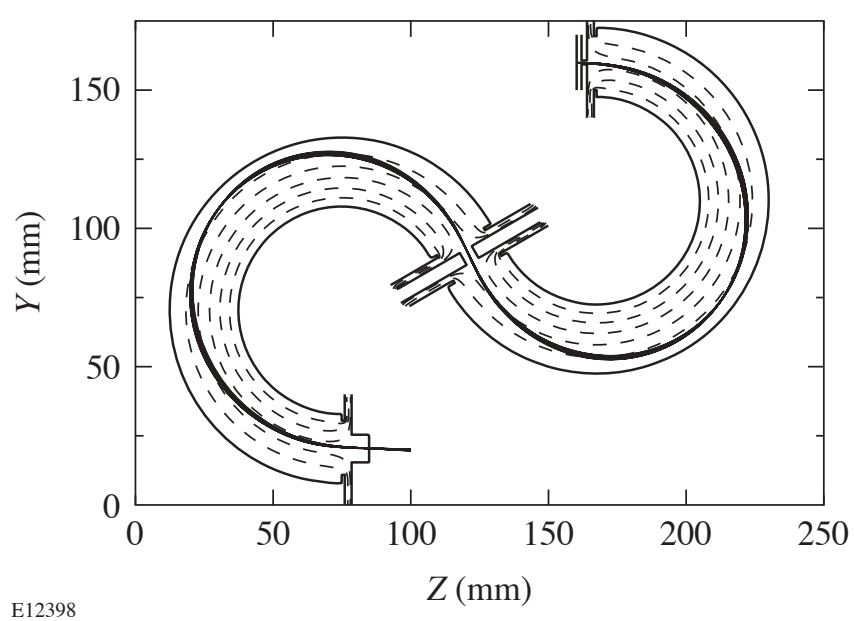

Figure 8: Double cylindrical analyzer is implemented as the front end of a femtosecond streak tube. Cylinder potentials are $V(37.5 \mathrm{~mm})=10.5 \mathrm{kV}, V(62.5 \mathrm{~mm})=4.285 \mathrm{kV}$. The beam potential is $7 \mathrm{kV}$. Cylinder height (out of the page) is greater than $100 \mathrm{~mm}$ to ensure a uniform gradient in the central region. The extraction field at the photocathode is $7 \mathrm{kV} / 1.75 \mathrm{~mm}$, which reduces to about $5.6 \mathrm{kV} / 1.75 \mathrm{~mm}$ opposite the slot.

potential gradient. As was shown previously with the deflection plates, this causes a temporal shear across the $y$ direction of the beam. Its magnitude is about $10 \mathrm{ps} / \mathrm{mm}$ of photocathode/cylinder for the present example. The second cylinder is oriented so that the temporal shear is effectively cancelled when the beam exits. The temporal shear for the double cylinders is reduced to $0.5 \mathrm{ps} / \mathrm{mm}$ of cathode for this design. The $T_{1 y}$ term is 0.232 , independent of cathode position, and there is no good way to restrict $\varepsilon_{y}$ to obtain femtosecond performance. A crossover point would be needed in the final imaging stage before the deflection system.

When a slot is used for the extraction electrode, the virtual photocathode is the transverse velocity distribution of the electrons emitted from the cathode. Thus at each image plane, one can use an aperture to restrict the transverse energy of the electrons. As with the mesh, there is a residual temporal shear on the beam as a function of transverse photocathode position. With a slot extraction electrode, however, the temporal shear goes through a minimum at an off-axis position. Fortunately, the $T_{1 y}$ term changes sign, going through zero, near the same position. The usable photocathode with $\left(T_{1 y}<\right.$ 0.05 ) is about $30 \mu \mathrm{m}$ for a $20 \mathrm{fs}$ contribution. A $25 \mu \mathrm{m}$ aperture is used at the exit image plane of the double cylinder to restrict $\varepsilon_{y}<25 \mathrm{meV}$. Aligning this aperture is a nontrivial mechanical task. The system time resolution is plotted in Fig. 9; FWHM is $40 \mathrm{fs}$, half the signal arrives within $50 \mathrm{fs}$, and the signal level at $100 \mathrm{fs}$ is 0.2 .

The problem of imaging the $X$ and $Y$ object planes, which are $50 \mathrm{~cm}$ apart, simultaneously to the screen can be readily solved with a quadrupole doublet. Magnifications are in the range of 0.5 to 1.0. This will increase the beam divergence to greater than that required for the deflection system. An aperture may be installed to restrict the beam divergence but then the throughput will be very small (few percent). An alternative that does work is the bilamellar tube, but using the F1 electrode to focus in the time direction as presented in Fig. 10. Here we obtain $M_{x}=0.2$ and $M_{y}=8.5$. The time-of-flight dispersion term, $\tau_{\varepsilon x}= \pm 1.3 \times X_{0} \times \sqrt{\varepsilon_{x}}+4.7 \times \varepsilon_{x}$, is too large and will dominate the system time resolution. Therefore we propose that there be no focusing in the $x$ direction and allow the beam from a single point to expand to $>10-\mathrm{mm}$ size during the 15-ns transit to the screen. Forgoing all spatial resolution is not unreasonable given the limited number of electrons that can be transported to the screen in a 50-fs-time-resolution element.

Space-charge broadening is a major concern for femtosecond diagnostics. A simple calculation using $1 \%$ of the ChildLangmuir current density limit at the photocathode $\left(0.01 \times 32 \mathrm{~A} / \mathrm{cm}^{2}\right)$ emitted from a $2-\mathrm{cm} \times 30-\mu \mathrm{m}$ cathode area in $50 \mathrm{fs}$, and assuming throughput values of $2 / 3$ for $X$ and 0.25 for $Y$, results in 100 electrons per time-resolution element. Given the relatively large area of cathode that is used, this is feasible. ${ }^{27,28}$ There is one caveat, a $\mathrm{KBr}$ photocathode has a $50 \%$ probability of emitting more than one secondary electron per absorbed $\mathrm{x}$ ray. (We have measured a $1.4 \%$ probability of ten or more secondary electrons per absorbed $\mathrm{x}$ ray.) This would of course result in spatial clumping of the emitted electrons. As an alternative, gold photocathodes emit an average of 1.16 secondary electrons per $\mathrm{x}$ ray. 

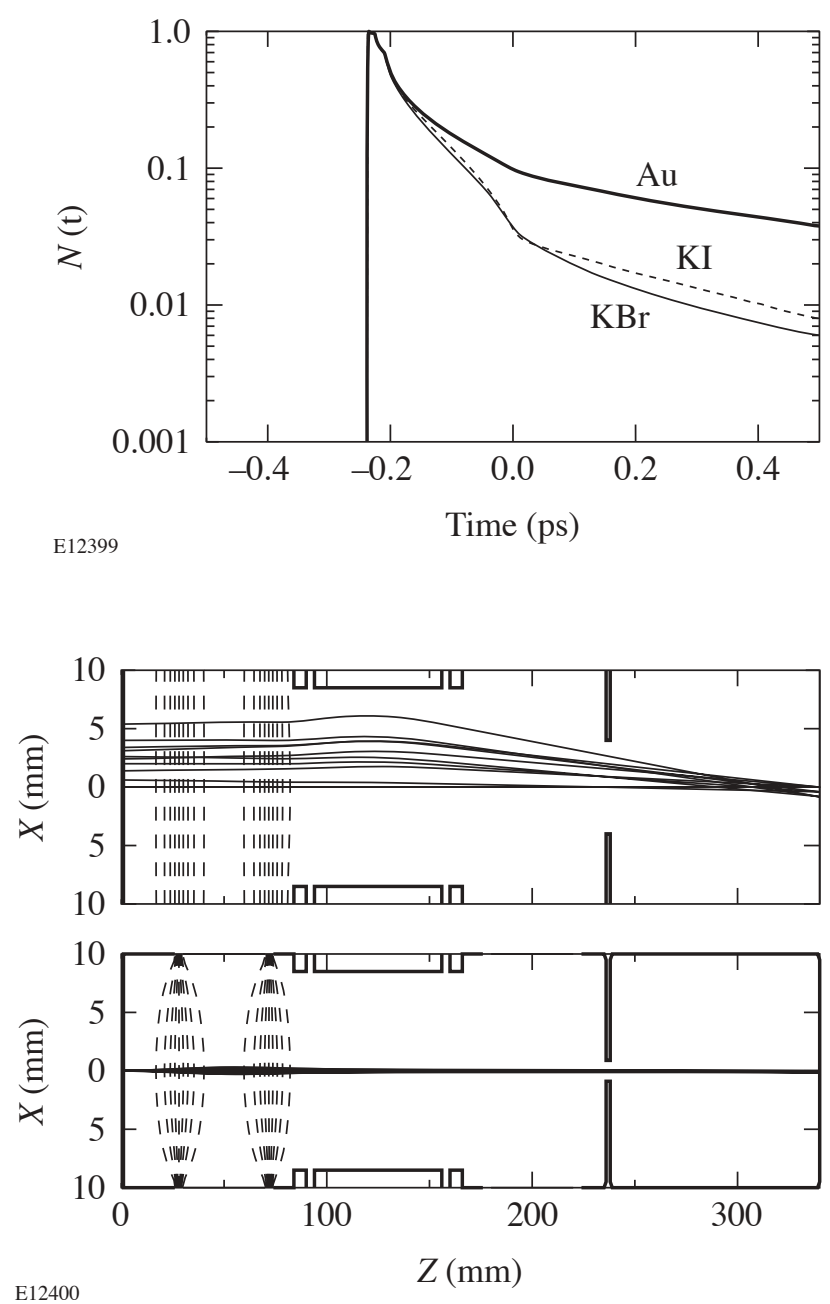

Figure 9: The time system response for the double cylinder calculated for $\mathrm{KI}, \mathrm{KBr}$, and $\mathrm{Au}$ X-ray photocathode materials. The $\varepsilon_{y}$ limit was set to $25 \mathrm{meV}$, and the time resolution obtained is $40 \mathrm{fs}$.

Figure 10: The output from the double cylinder is imaged to the screen with a bilamellar tube. The spatial magnification is 0.2 , with the crossover point just in front of the screen. The $y$-direction magnification is 8.5 , which reduces the beam divergence to that required by the deflection system.

\section{CONCLUSIONS}

We have presented a novel streak tube design that clearly has the potential for demonstrating 100-fs time resolution. It utilizes a combination of a modest extraction field at the photocathode, axial time-of-flight dispersion compensation, and transverse energy selection. Increasing the extraction field and/or the cylinder radii will allow the beam energy in the double cylinder to be increased if space charge is a concern. Imaging the spatial dimension with negligible dispersion remains an issue and would be highly desired for cameras operated in accumulating mode (nominal 1-kHz repetition rate) with a $<50$-fs jitter streak ramp. ${ }^{15}$ Interestingly, the time resolution does not change significantly with $\mathrm{x}$-ray photocathode material. In fact, the narrower energy distributions generated off optical photocathodes are more difficult to compensate. This design can also be applied to time-resolved electron diffraction experiments that use a photocathode as an electron source. ${ }^{27}$

\section{ACKNOWLEDGMENT}

This work was supported by the U.S. Department of Energy Office of Inertial Confinement Fusion under Cooperative Agreement No. DE-FC03-92SF19460, the University of Rochester, and the New York State Energy Research and Development Authority. The support of DOE does not constitute an endorsement by DOE of the views expressed in this article. 


\section{REFERENCES}

1. J. S. Courtney-Pratt, "A new method for the photographic study of fast transient phenomena," Research 2, 287-294 (1949).

2. D. J. Bradley, "Electron-optical image tubes and image tube streak cameras," U.S. Patent No. 3,761,614 (25 September 1973); ibid., U.K. Patent No. 1,329,977 (25 September 1973).

3. D. J. Bradley and W. Sibbett, "Subpicosecond chronoscopy," Appl. Phys. Lett. 27(7), 382-384 (1975).

4. K. Kinoshita, M. Ito, and Y. Suzuki, "Femtosecond streak tube," Rev. Sci. Instrum. 58(6), 932-938 (1987).

5. H.-B. Niu, H. Zhang, Q. L. Yang, Y. P. Liu, Y. C. Wang, Y. A. Reng, and J.-H. Zhou, “Theoretical and experimental study of femtosecond streak image tube," in 18th International Congress on High Speed Photography and Photonics, edited by D.-H. Wang, Vol. 1032, pp. 472-481, SPIE, Bellingham, WA, 1989.

6. A. Finch, Y. P. Liu, H.-B. Niu, W. Sibbett, W. E. Sleat, D. R. Walker, Q. L. Yang, and H. Zhang, "Development and evaluation of a new femtosecond streak camera," in 18th International Congress on High Speed Photography and Photonics, edited by D.-H. Wang, Vol. 1032, pp. 622-627, SPIE, Bellingham, WA, 1989.

7. A. J. Lieber, H. D. Sutphin, C. B. Webb, and A. H. Williams, "Developing a subpicosecond streak camera," Electro-Opt. Syst. Des. 8(9), 26-29 (1976).

8. An excellent review of the progress in optical and x-ray streak cameras can be found in the Proceedings of the International Congress on High-Speed Photography and Photonics, a biennial meeting. See also the Proceedings of the High Speed Photography, Videography, and Photonics, San Diego (1983-1988) and Ultrahigh- and High-Speed Photography, Videography, and Photonics, San Diego (1989-1993).

9. M. M. Murnane, H. C. Kapteyn, and R. W. Falcone, "X-ray streak camera with 2 ps response," Appl. Phys. Lett. 56(20), 1948-1950 (1990).

10. Z. Chang, A. Rundquist, J. Zhou, M. M. Murnane, H. C. Kapteyn, X. Liu, B. Shan, J. Liu, L. Niu, M. Gong, and X. Zhang, "Demonstration of a sub-picosecond x-ray streak camera," Appl. Phys. Lett. 69(1), 133-135 (1996).

11. C. Y. Cote, J.-C. Kieffer, P. Gallant, J.-C. Rebuffie, C. Goulmy, A. M. Maksimchuk, G. A. Mourou, D. Kaplan, and M. Bouvier, "Development of a subpicosecond large-dynamic-range x-ray streak camera," in 22nd International Congress High-Speed Photography and Photonics, edited by D. L. Paisley, Vol. 2869, pp. 956-961, SPIE, Bellingham, WA, 1997. 12. P. Gallant, P. Forget, F. Dorchies, Z. Jiang, J. C. Kieffer, P. A. Jaanimagi, J. C. Rebuffie, C. Goulmy, J. F. Pelletier, and M. Sutton, "Characterization of a subpicosecond x-ray streak camera for ultrashort laser-produced plasmas experiments," Rev. Sci. Instrum. 71(10), 3627-3633 (2000).

13. G. A. Naylor, K. Scheidt, J. Larsson, M. Wulff, and M. Filhol, "A sub-picosecond accumulating streak camera for x-rays," Meas. Sci. Technol. 12(11), 1858-1864 (2001).

14. C. Belzile, J. C. Kieffer, C. Y. Cote, T. Oksenhendler, and D. Kaplan, "Jitter-free subpicosecond streak cameras," Rev. Sci. Instrum. 73(3), 1617-1620 (2002).

15. J. Liu, J. Wang, B. Shan, C. Wang, and Z. Chang, “An accumulative x-ray streak camera with sub-600-fs temporal resolution and 50-fs timing jitter,” Appl. Phys. Lett. 82(20), 3553-3555 (2003).

16. E. K. Zavoisky and S. D. Fanchenki, "Image converter high-speed photography with $10^{-9}-10^{-14}$ sec time resolution," Appl. Opt. 4(9), 1155-1167 (1965).

17. P. A. Jaanimagi, A. Mens, and J.-C. Rebuffie, "Photoelectron throughput in streak tubes," in Ultrahigh-and High-Speed Photography, Videography, and Photonics '95, edited by G. A. Kyrala and D. R. Snyder, Vol. 2549, pp. 62-71, SPIE, Bellingham, WA, 1995.

18. K. Kinoshita, "Streak tube having an arrangement for suppressing travel time spread of photoelectrons," U.S. Patent No. 5,221,836 (22 June 1993).

19. B. L. Henke, J. Liesegang, and S. D. Smith, "Soft-x-ray-induced secondary-electron emission from semiconductors and insulators: Models and measurements," Phys. Rev. B, Condens. Matter 19(6), 3004-3021 (1979).

20. G. Clément, C. Loty, J. P. Roux, and C. Chancel, "The design of a new electron optics for a picosecond streak camera," in Proceedings of the Eleventh International Congress on High Speed Photography, edited by P. J. Rolls, pp. 130-135, Chapman and Hall, London, 1975. 
21. A. Mens, D. Gontier, J.-C. Huilizen, R. Sauneuf, D. Schirmann, R. Verrecchia, J.-P. Chambaret, G. Hamoniaux, J. M. Roth, and F. Tomasini, "New developments and results on high-spatiotemporal-resolution x-ray streak cameras," in Ultrahigh- and High-Speed Photography, Videography, and Photonics '91, edited by P. A. Jaanimagi, Vol. 1539, pp. 40-51, SPIE, Bellingham, WA, 1992 and references therein.

22. H. D. Sutphin and A. J. Lieber, "Proximity focused streak tube and streak camera using the same," U.S. Patent No. 4,266,247 (5 May 1981).

23. H.-B. Niu, V. P. Degtyareva, V. N. Platonov, A. M. Prokhorov, and M. Y. Schelev, "Specially designed femtosecond streak image tube with temporal resolution of $50 \mathrm{fs,"} \mathrm{in} \mathrm{18th} \mathrm{International} \mathrm{Congress} \mathrm{on} \mathrm{High} \mathrm{Speed} \mathrm{Photography} \mathrm{and}$ Photonics, edited by D.-H. Wang, Vol. 1032, pp. 79-86, SPIE, Bellingham, WA, 1989.

24. S. Okayama, "A new type of quadrupole correction lens for electron-beam lithography," Nucl. Instrum. Methods Phys. Res. A A298(1-3), 488-495 (1990).

25. K. Kinoshita, T. Kato, and Y. Suzuki, "The electron trajectory analysis in the deflecting electric field of the streak tube," in Proceedings of the 14th International Congress on High Speed Photography and Photonics, edited by B. M. Stepanov, pp. 199-202, Society of Photo-Optical Instrumentation, Bellingham, WA, 1980.

26. G. F. Rempfer, "A theoretical study of the hyperbolic electron mirror as a correcting element for spherical and chromatic aberration in electron optics," J. Appl. Phys. 67(10), 6027-6040 (1990).

27. B.-L. Quian and H. E. Elsayed-Ali, "Electron pulse broadening due to space charge effects in a photoelectron gun for electron diffraction and streak camera systems," J. Appl. Phys. 91(1), 462-468 (2002).

28. B. J. Siwick, J. R. Dwyer, R. E. Jordan, and R. J. D. Miller, "Ultrafast electron optics: Propagation dynamics of femtosecond electron packets," J. Appl. Phys. 92(3), 1643-1648 (2002). 